\title{
THE MINIMAL ENTROPY MARTINGALE MEASURE FOR EXPONENTIAL MARKOV CHAINS
}

\author{
YOUNG LEE,* Deutsche Bank AG and London School of Economics \\ THORSTEN RHEINLÄNDER, ** Vienna University of Technology and \\ London School of Economics
}

\begin{abstract}
In this article we investigate the minimal entropy martingale measure for continuous-time Markov chains. The conditions for absence of arbitrage and existence of the minimal entropy martingale measure are discussed. Under this measure, expressions for the transition intensities are obtained. Differential equations for the arbitrage-free price are derived.

Keywords: Continuous-time Markov chain; relative entropy; martingale measure

2010 Mathematics Subject Classification: Primary 60J25

Secondary 91B28
\end{abstract}

\section{Introduction}

The main contribution of this paper is the calculation of the minimal entropy martingale measure for continuous-time Markov chains as explicitly as possible in terms of the transition intensities.

Many different types of model have been proposed to describe financial asset prices. In particular, the dynamics of stock prices have been modelled as exponential Lévy processes, such as in [4] and [15], while another popular model class consists of diffusions with stochastic volatility; see, e.g. [10]. More complex models for the price process comprise the general Barndorff-Nielsen and Shephard model where both the price and volatility processes contain jump terms which are allowed to correlate with one another; see [20]. On another spectrum, the modelling of asset returns by means of pure jump processes have been studied by [1], [3], and [17], to mention a few.

In this study we employ the continuous-time Markov chain model proposed in [17] while making the minor modification of relaxing the assumption of constant 'drift' and 'volatility' parameters by allowing for time dependency. Markov chains are relatively easy to implement numerically while giving a reasonable fit to empirically observed asset prices; see [17] and [18].

Such models typically generate incomplete markets, which means that there exist infinitely many martingale measures which are equivalent to the physical measure describing the trajectories of the underlying price process. Each equivalent martingale measure corresponds to a set of derivative prices attuned with the no-arbitrage constraint. Many different techniques to the problem of identifying an appropriate equivalent martingale measure for derivative pricing

\footnotetext{
Received 27 May 2008; revision received 17 September 2012.

* Postal address: Deutsche Bank AG, 1 Great Winchester Street, London EC2N 2DB, UK.

Email address: young.lee@db.com

The views expressed in this paper are those of the author and do not necessarily reflect the position of Deutsche Bank AG.

** Postal address: Financial and Actuarial Mathematics, Vienna University of Technology, Wiedner Hauptstraße 8/105-1, 1040 Vienna, Austria. Email address: rheinlan@fam.tuwien.ac.at
} 
have been proposed in the literature, but there is not yet an ultimate and definitive way of selecting any one of them.

One methodology to find an adequate equivalent martingale measure consists in employing a dual approach to associate it with a utility function describing the investors' preferences. It is well known that maximizing expected utility admits a dual formulation in the sense that finding an equivalent martingale measure corresponds to minimizing some sort of distance to the physical probability measure. For investors having an exponential utility function, the dual problem is the minimization of relative entropy [5].

The minimal entropy martingale measure for Lévy processes is well studied. Hubalek and Sgarra [9] give an excellent review. The minimal entropy martingale measure for BarndorffNielsen and Shephard models have also been investigated in [2] and [20]. More recently, Lee and Rheinländer [13] discussed the entropic measure for a defaultable asset driven by both a Brownian motion and the counting process martingale associated to the one-jump process. However, the minimal entropy martingale measure for continuous-time Markov chains has not been studied so far. Miyahara [16] examined the minimal entropy martingale measure for a birth-and-death process by means of the Hamilton-Jacobi-Bellman equation. In this paper we extend to the case where the asset price process is modelled by a semimartingale with the dynamics of the risky asset following a continuous-time Markov chain.

Our method of computing the minimal entropy martingale measure for continuous-time Markov chains follows the approaches and procedures outlined in [5] and [8].

In Section 2 we summarize the basic definitions and main results concerning continuoustime Markov chains. In Section 3 we present our asset model as well as the basic properties of relative entropy. In Sections 4 and 5 we explain our martingale approach setup to finding the minimal entropy martingale measure. We then fix an asset price process for which we further our analysis to finding a candidate for the minimal entropy martingale measure by means of the work in earlier sections. The conditions for absence of arbitrage and existence of solutions are discussed. In Sections 6 and 7 we present our main idea. In Section 9 we discuss the pricing of derivatives under the minimal entropy martingale measure. This section follows the spirit of [17] and our aim is to derive the integrodifferential equations when the derivative prices are evaluated under the minimal entropy martingale measure.

\section{Continuous-time Markov chains}

The mathematical framework is given by a filtered probability space $(\Omega, \mathcal{F}, \mathbb{F}, \mathbb{P})$ and a finite time horizon $T<\infty$. We assume that $\mathcal{F}_{0}$ is trivial and that $\mathcal{F}=\mathcal{F}_{T}$. Let $\left\{C_{t}\right\}_{0 \leq t \leq T}$ be a continuous-time $(\mathbb{F}, \mathbb{P})$-Markov chain in the finite state space $\mathcal{y}=\{1, \ldots, m\}$. Furthermore, let $\mathbb{F}$ be the completion of the filtration $\mathbb{F} C=\left(\mathcal{F}_{t}^{C}\right)_{0 \leq t \leq T}=\sigma\left(C_{s} ; 0 \leq s \leq t\right), 0 \leq t \leq T$, generated by this Markov chain such that $(\Omega, \mathcal{F}, \mathbb{F}, \mathbb{P})$ becomes a complete filtered probability space. The paths of $C$ are taken to be right continuous and $C_{0}$ is taken to be deterministic. Assume further that $C$ is time homogeneous so that we have

$$
\mathbb{P}\left(C_{t+s}=j \mid C_{s}=i\right)=p_{i j}(t) \quad \text { for all } i, j \in \mathcal{Y}, s, t \in \mathbb{R}^{+}, 0 \leq s \leq t \leq T .
$$

The following limit exists for $i, j \in \mathcal{y}$ (see Theorem 8.1.2 of [23]):

$$
\mu^{i j}:=\lim _{t \searrow 0} \frac{p_{i j}(t)-p_{i j}(0)}{t} .
$$

We note that, for every $i \neq j$, we have $\mu^{i j} \geq 0$ and $\mu^{i i}=-\sum_{j=1, j \neq i}^{m} \mu^{i j}$. Hence, $\mu^{i j}$ represents the intensity of transition from state $i$ to state $j$ and is a constant. The matrix 
$\Lambda:=\left[\mu^{i j}\right]_{i, j \in\{1, \ldots, m\}}$ is called the infinitesimal generator matrix for a Markov chain. It is also commonly known as the intensity matrix. We say that a state $i \in \mathcal{y}$ is absorbing for a time-homogeneous $(\mathbb{F}, \mathbb{P})$-Markov chain $C_{t}$, where $t \in \mathbb{R}^{+}$, if the following holds:

$$
\mathbb{P}\left(C_{t}=i \mid C_{s}=i\right)=1 \text { for all } s, t \in \mathbb{R}^{+}, s \leq t \leq T .
$$

It is clear that if a state $i \in \mathcal{Y}$ is absorbing then we have $\mu^{i j}=0$ for every $j=1, \ldots, m$. We assume throughout that there are no absorbing states. Furthermore, we assume that there are at least three distinct states, i.e. $m \geq 3$, so the market under consideration is incomplete. We introduce, for $i \neq j$ and all $s, t \in \mathbb{R}^{+}, t \leq T$,

$$
\begin{aligned}
H_{t}^{i} & :=\mathbf{1}\left\{C_{t}=i\right\}, \\
H_{t}^{i j} & :=\sharp\left\{s: 0<s \leq t ; C_{s-}=i, C_{s}=j\right\}=\sum_{0<s \leq t} \mathbf{1}\left\{C_{s-}=i\right\} \mathbf{1}\left\{C_{s}=j\right\} .
\end{aligned}
$$

Thus, $H_{t}^{i}$ is the indicator of the event that $C$ is in state $i$ at time $t$. On the other hand, $H_{t}^{i j}$ is the number of jumps from $i$ to $j$ during $(0, t]$. We state some results from [12] and [22].

Lemma 1. For every $i, j \in \mathcal{Y}, i \neq j$, the processes

$$
U_{t}^{i j}=H_{t}^{i j}-\int_{0}^{t} \mu^{i j} H_{u}^{i} \mathrm{~d} u
$$

are $(\mathbb{P}, \mathbb{F})$-martingales.

Proof. See Theorem 7.5.5 of [12].

Theorem 1. Any arbitrary $(\mathbb{P}, \mathbb{F})$-local martingale $M$ can be written as

$$
M_{t}=\int_{0}^{t} \sum_{i} \sum_{j} g_{s}(i, j) \mathrm{d} U_{s}^{i j},
$$

where $g$ is locally bounded and predictable for all $i, j \in \mathcal{y}$ and $g(i, i)=0$ for all $i \in \mathcal{Y}$, $0 \leq t \leq T$.

Proof. See Lemma 21.13 and Theorem 21.15 of [22].

\section{Asset model and general results}

Our choice of asset price process $X$ under $\mathbb{P}$ is inspired by the asset price process introduced in [17], i.e.

$$
\frac{\mathrm{d} X_{t}}{X_{t-}}=\sum_{i} \eta_{t}^{i} H_{t}^{i} \mathrm{~d} t+\sum_{i} \sum_{j} \theta_{t}^{i j} \mathrm{~d} U_{t}^{i j},
$$

where $\eta_{t}^{i}$ and $\theta_{t}^{i j}>-1$ are deterministic and bounded functions for a fixed $i \in \mathcal{y}$. It follows that $X$ has the decomposition $X=X_{0}+M+A$, where $M$ is a local martingale null at zero and $A$ is a process with finite variation. By Theorem 1, we may write $M$ as

$$
M=\int \sum_{i} \sum_{j}{ }^{M} \widetilde{\varphi}^{i j} \mathrm{~d} U^{i j},
$$

where the ${ }^{M} \widetilde{\varphi}^{i j}, i, j \in \mathcal{Y}, i \neq j$, are bounded $\mathbb{F}$-predictable with ${ }^{M} \widetilde{\varphi}^{i i}=0$ for $i=1, \ldots, m$. Moreover, we assume that the asset price process $X$ satisfies the structure condition (see Definition 2.39 of [21]): there exists a predictable process $\lambda$ satisfying $A=\int \lambda \mathrm{d}\langle M, M\rangle$, with $K_{T}:=\int_{0}^{T} \lambda_{s}^{2} \mathrm{~d}\langle M, M\rangle_{s}<\infty, \mathbb{P}$-almost surely ( $\mathbb{P}$-a.s.). 
Definition 1. Let $\mathcal{V}$ be the linear subspace of $L^{\infty}(\Omega, \mathcal{F}, \mathbb{P})$ spanned by the elementary stochastic integrals of the form $f=h\left(X_{T_{2}}-X_{T_{1}}\right)$, where $0 \leq T_{1} \leq T_{2} \leq T$, are stopping times such that the stopped process $X^{T_{2}}$ is bounded and $h$ is a bounded $\mathcal{F}_{T_{1}}$-measurable random variable. A martingale measure is a probability measure $\mathbb{Q} \ll \mathbb{P}$ with $\mathbb{E}_{\mathbb{P}}[(\mathrm{d} \mathbb{Q} / \mathrm{d} \mathbb{P}) f]=0$ for all $f \in \mathcal{V}$.

The sets of absolutely continuous martingale measures and equivalent martingale measures for $X$ with respect to $\mathbb{F}$ are defined as

$$
\begin{aligned}
\mathcal{M} & :=\{\mathbb{Q} \ll \mathbb{P} \mid X \text { is a local }(\mathbb{Q}, \mathbb{F}) \text {-martingale }\}, \\
\mathcal{M}^{\mathrm{e}}: & =\{\mathbb{Q} \sim \mathbb{P} \mid X \text { is a local }(\mathbb{Q}, \mathbb{F}) \text {-martingale }\} .
\end{aligned}
$$

Note that, as $X$ is locally bounded, a probability measure $\mathbb{Q}$ absolutely continuous to $\mathbb{P}$ is in $\mathcal{M}$ if and only if $X$ is a local $\mathbb{Q}$-martingale.

Definition 2. The relative entropy $H(\mathbb{Q}, \mathbb{P})$ of a probability measure $\mathbb{Q}$ with respect to a probability measure $\mathbb{P}$ is given as

$$
H(\mathbb{Q}, \mathbb{P})= \begin{cases}\mathbb{E}_{\mathbb{P}}\left[\frac{\mathrm{d} \mathbb{Q}}{\mathrm{d} \mathbb{P}} \log \frac{\mathrm{d} \mathbb{Q}}{\mathrm{d} \mathbb{P}}\right] & \text { if } \mathbb{Q} \ll \mathbb{P}, \\ +\infty & \text { otherwise }\end{cases}
$$

It is well known that $H(\mathbb{Q}, \mathbb{P}) \geq 0, H(\mathbb{Q}, \mathbb{P})=0$ if and only if $\mathbb{Q}=\mathbb{P}$, and $\mathbb{Q} \rightarrow H(\mathbb{Q}, \mathbb{P})$ is strictly convex.

Definition 3. The minimal entropy martingale measure $\mathbb{Q}^{E}$ is the solution of

$$
H\left(\mathbb{Q}^{E}, \mathbb{P}\right)=\min _{\mathbb{Q} \in \mathcal{M}} H(\mathbb{Q}, \mathbb{P}) .
$$

Theorems 1 and 2 and Remark 1 of [7] as well as the fact that $\mathcal{V} \subset L^{\infty}(\mathbb{P})$ yield the following result from [7].

Theorem 2. If there exists $\mathbb{Q} \in \mathcal{M}^{\mathrm{e}}$ such that $H(\mathbb{Q}, \mathbb{P})<\infty$, then the minimal entropy martingale measure exists, is unique, and, moreover, equivalent to $\mathbb{P}$.

We now state a theorem from [8] which provides a criterion for a martingale measure to coincide with the minimal entropy martingale measure.

Theorem 3. Assume that there exists a $\mathbb{Q} \in \mathcal{M}^{\mathrm{e}}$ with $H(\mathbb{Q}, \mathbb{P})<\infty$. Then $\overline{\mathbb{Q}}$ is the minimal entropy martingale measure if and only if there exists a constant $c^{E}$ and an $X$-integrable predictable process $\phi^{E}$,

$$
\frac{\mathrm{d} \overline{\mathbb{Q}}}{\mathrm{d} \mathbb{P}}=\exp \left(c^{E}+\int_{0}^{T} \phi_{t}^{E} \mathrm{~d} X_{t}\right),
$$

such that $\mathbb{E}_{\mathbb{Q}}\left[\int_{0}^{T} \phi_{t}^{E} \mathrm{~d} X_{t}\right]=0$ for all $\mathbb{Q} \in \mathcal{M}^{\mathrm{e}}$ with finite relative entropy.

Our strategy would then be to pursue finding some potential candidate measure $\overline{\mathbb{Q}}$ which can be represented as in (2). We would then verify that this potential measure $\overline{\mathbb{Q}}$ is indeed the entropy minimizer. To carry out this last step, we appeal to the verification procedures of Theorem 2.1.5 of [8]. 


\subsection{Verification procedures}

Let us now describe the procedures in full, consisting of four steps, for verifying that a given probability measure is indeed the minimal entropy martingale measure $\mathbb{Q}^{E}$.

Step 1: $\overline{\mathbb{Q}}$ is an equivalent probability measure. To demonstrate this, we have to show that $\exp \left\{c^{E}+\int_{0}^{T} \phi_{t}^{E} \mathrm{~d} X_{t}\right\}$ is integrable with

$$
\mathbb{E}_{\mathbb{P}}\left[\exp \left\{c^{E}+\int_{0}^{T} \phi_{t}^{E} \mathrm{~d} X_{t}\right\}\right]=1 .
$$

Step 2: $\overline{\mathbb{Q}}$ is a martingale measure, i.e. $X$ is a local $\overline{\mathbb{Q}}$-martingale.

Step 3: The probability measure $\overline{\mathbb{Q}}$ has finite relative entropy with respect to $\mathbb{P}$, i.e.

$$
H(\overline{\mathbb{Q}}, \mathbb{P})=\mathbb{E}_{\mathbb{P}}\left[\frac{\mathrm{d} \overline{\mathbb{Q}}}{\mathrm{d} \mathbb{P}} \log \frac{\mathrm{d} \overline{\mathbb{Q}}}{\mathrm{d} \mathbb{P}}\right]=\mathbb{E}_{\overline{\mathbb{Q}}}\left[\log \frac{\mathrm{d} \overline{\mathbb{Q}}}{\mathrm{d} \mathbb{P}}\right]<\infty .
$$

Step 4: $\int \phi^{E} \mathrm{~d} X$ is a true $\mathbb{Q}$-martingale for all $\mathbb{Q} \in \mathcal{M}^{\mathrm{e}}$ with $H(\mathbb{Q}, \mathbb{P})<\infty$.

If all of these conditions are satisfied then $\overline{\mathbb{Q}}$ is the minimal entropy martingale measure $\mathbb{Q}^{E}$.

\section{Equivalent martingale measures for continuous-time Markov chains}

A probability measure $\mathbb{Q} \ll \mathbb{P}$ on the filtered probability space $(\Omega, \mathcal{F}, \mathbb{F}, \mathbb{P})$ is a martingale measure for $X$ if $X$ is a local $\mathbb{Q}$-martingale. From Theorem 2.42 of [21], we state a result which ensures that $\mathbb{Q}$ is a martingale measure.

Corollary 1. (Equivalent martingale measure.) Let $\mathbb{Q}$ be a probability measure whose density process $Z:=\mathrm{d} \mathbb{Q} / \mathrm{d} \mathbb{P}$ is given by the Doléans Dade exponential process

$$
Z=\mathcal{E}\left(-\int \lambda \mathrm{d} M+L\right)
$$

where $L$ and $[M, L]$ are locally bounded local $\mathbb{P}$-martingales. Then the probability measure $\mathbb{Q}$ is a martingale measure.

Since $L$ is a local martingale we may write

$$
L=\int \sum_{i} \sum_{j}{ }^{L} \widetilde{\varphi}^{i j} \mathrm{~d} U^{i j}
$$

owing to Theorem 1. Furthermore, we have the following result from [24].

Proposition 1. Let $\mathbb{Q} \in \mathcal{M}^{\mathrm{e}}$. Let $1-\lambda_{t}^{M} \widetilde{\varphi}_{t}^{i j}+{ }^{L} \widetilde{\varphi}_{t}^{i j}>0$. Then the density process $Z:=\mathrm{d} \mathbb{Q} / \mathrm{d} \mathbb{P}$ is given by the Doléans-Dade exponential process

$$
Z=\varepsilon\left(-\int \lambda \mathrm{d} M+L\right)
$$

where $L$ and $[M, L]$ are local $\mathbb{P}$-martingales.

We now state some initial results. 
Proposition 2. Let $\mathbb{Q} \in \mathcal{M}^{\mathrm{e}}$. Then $Z_{T}=\mathcal{E}\left(-\int \lambda \mathrm{d} M+L\right)_{T}$ is given explicitly as

$$
\begin{aligned}
Z_{T}= & \exp \left\{\int_{0}^{T} \sum_{i} \sum_{j}\left(\lambda_{t}^{M} \widetilde{\varphi}_{t}^{i j}-{ }^{L} \widetilde{\varphi}_{t}^{i j}\right) \mu^{i j} H_{t}^{i} \mathrm{~d} t\right\} \\
& \left.\times \prod_{i} \prod_{\substack{j \neq j \\
0<t \leq T}} \prod_{0<t \leq}\left(1+\lambda_{t}^{M} \widetilde{\varphi}_{t}^{i j}+{ }^{L} \widetilde{\varphi}_{t}^{i j}\right) \Delta H_{t}^{i j}\right) .
\end{aligned}
$$

Proof. Apply Itô's formula to $\log Z$ :

$$
\begin{aligned}
\log Z_{T}= & \int_{0}^{T} \frac{1}{Z_{t-}} \mathrm{d} Z_{t}-\frac{1}{2} \int_{0}^{T} \frac{1}{Z_{t-}^{2}} \mathrm{~d}\langle Z, Z\rangle_{t}^{c}+\sum_{0<t \leq T}\left\{\log \frac{Z_{t}}{Z_{t-}}-\frac{1}{Z_{t-}} \Delta Z_{t}\right\} \\
= & \int_{0}^{T} \sum_{i} \sum_{j}\left(\lambda_{t}^{M} \widetilde{\varphi}_{t}^{i j}-{ }^{L} \widetilde{\varphi}_{t}^{i j}\right) \mu^{i j} H_{t}^{i} \mathrm{~d} t \\
& +\sum_{0<t \leq T}\left\{\log \left(1+\sum_{i} \sum_{j}\left(-\lambda_{t}^{M} \widetilde{\varphi}_{t}^{i j}+{ }^{L} \widetilde{\varphi}_{t}^{i j}\right) \Delta H_{t}^{i j}\right)\right\} .
\end{aligned}
$$

Also, $Z_{t}=\mathcal{E}(N)_{t}$ and, thus, $\mathrm{d} Z_{t}=Z_{t-} \mathrm{d} N_{t}$, so $\Delta Z_{t}=Z_{t-} \Delta N_{t}$. Also, note that $Z_{t} / Z_{t-}=$ $1+\Delta N_{t}$. Taking the exponential of both sides yields

$$
\begin{aligned}
Z_{T}= & \exp \left\{\int_{0}^{T} \sum_{i} \sum_{j}\left(\lambda_{t}^{M} \widetilde{\varphi}_{t}^{i j}-{ }^{L} \tilde{\varphi}_{t}^{i j}\right) \mu^{i j} H_{t}^{i} \mathrm{~d} t\right\} \\
& \times \prod_{0<t \leq T}\left(1+\sum_{i} \sum_{j}\left(-\lambda_{t}^{M} \widetilde{\varphi}_{t}^{i j}+{ }^{L} \widetilde{\varphi}_{t}^{i j}\right) \Delta H_{t}^{i j}\right) \\
= & \exp \left\{\int_{0}^{T} \sum_{i} \sum_{j}\left(\lambda_{t}^{M} \widetilde{\varphi}_{t}^{i j}-{ }^{L} \widetilde{\varphi}_{t}^{i j}\right) \mu^{i j} H_{t}^{i} \mathrm{~d} t\right\} \\
& \times \prod_{i} \prod_{j} \prod_{0<t \leq T}\left(1+\left(-\lambda_{t}^{M} \widetilde{\varphi}_{t}^{i j}+{ }^{L} \widetilde{\varphi}_{t}^{i j}\right) \Delta H_{t}^{i j}\right),
\end{aligned}
$$

where the last line follows from the fact that, for a fixed $t$, and every $i \neq j$ and $k \neq l,(i, j) \neq$ $(k, l)$, the processes $H_{t}^{i j}$ and $H_{t}^{k l}$ have no common jumps; see [22, Proof of Theorem 22.9].

Our approach now would be to find $L$ via ${ }^{L} \widetilde{\varphi}^{i j}$ for $i \neq j$ such that the corresponding martingale measure $\overline{\mathbb{Q}}$ has the form of (2) and then perform the verifications as outlined in Section 3.1.

\section{The entropy equation}

Theorem 4. The strategy $\phi^{E}$ and the constant $c^{E}$ in (2) satisfy the equation

$$
\begin{aligned}
& c^{E}+\int_{0}^{T} \sum_{i} \sum_{j} \phi_{t}^{E} \lambda_{t}\left({ }^{M} \widetilde{\varphi}_{t}^{i j}\right)^{2} \mu^{i j} H_{t}^{i} \mathrm{~d} t-\int_{0}^{T} \sum_{i} \sum_{j} \phi_{t}^{E M} \widetilde{\varphi}_{t}^{i j} \mu^{i j} H_{t}^{i} \mathrm{~d} t \\
& -\int_{0}^{T} \sum_{i} \sum_{j} \lambda_{t}{ }^{M} \widetilde{\varphi}_{t}^{i j} \mu^{i j} H_{t}^{i} \mathrm{~d} t+\int_{0}^{T} \sum_{i} \sum_{j}{ }^{L} \widetilde{\varphi}_{t}^{i j} \mu^{i j} H_{t}^{i} \mathrm{~d} t \\
& \quad=-\int_{0}^{T} \sum_{i} \sum_{j} \phi_{t}^{E M} \widetilde{\varphi}_{t}^{i j} \mathrm{~d} H_{t}^{i j}+\int_{0}^{T} \sum_{i} \sum_{j} \log \left(1-{ }^{M} \widetilde{\varphi}_{t}^{i j} \lambda_{t}+{ }^{L} \widetilde{\varphi}_{t}^{i j}\right) \mathrm{d} H_{t}^{i j},
\end{aligned}
$$


where, for every $i \neq j,{ }^{L} \widetilde{\varphi}_{t}^{i j}$ has to be chosen such that

$$
\sum_{i} \sum_{j}{ }^{M} \widetilde{\varphi}_{t}^{i j L} \widetilde{\varphi}_{t}^{i j} \mu^{i j} H_{t}^{i} \mathrm{~d} t=0 \quad \text { for all } t \in[0, T] .
$$

Proof. From Proposition 1, we see that $[M, L]$ is a local $\mathbb{P}$-martingale. Observe that

$$
[M, L]_{t}=\int_{0}^{t} \sum_{i} \sum_{j}{ }^{M} \tilde{\varphi}_{s}^{i j L} \widetilde{\varphi}_{s}^{i j} \mathrm{~d} H_{s}^{i j} .
$$

We further know from [6, Section VII.39] that the predictable bracket process

$$
\langle M, L\rangle_{t}=\int_{0}^{t} \sum_{i} \sum_{j}{ }^{M} \widetilde{\varphi}_{s}^{i j L} \widetilde{\varphi}_{s}^{i j} \mu^{i j} H_{s}^{i} \mathrm{~d} s
$$

exists, since $M$ and $L$ are locally bounded. However, $\langle M, L\rangle=0$ because $[M, L]$ is a local martingale. We therefore get (5). Recall that

$$
\mathrm{d} X_{t}=\sum_{i} \sum_{j}{ }^{M} \widetilde{\varphi}_{t}^{i j} \mathrm{~d} U_{t}^{i j}+\sum_{i} \sum_{j} \lambda_{t}\left({ }^{M} \widetilde{\varphi}_{t}^{i j}\right)^{2} \mu^{i j} H_{t}^{i} \mathrm{~d} t .
$$

By (2) and (3) we have

$$
\begin{aligned}
\log Z_{T} & =c^{E}+\int_{0}^{T} \phi_{t}^{E} \mathrm{~d} X_{t} \\
& =c^{E}+\int_{0}^{T} \sum_{i} \sum_{j} \phi_{t}^{E M} \widetilde{\varphi}_{t}^{i j} \mathrm{~d} U_{t}^{i j}+\int_{0}^{T} \sum_{i} \sum_{j} \lambda_{t} \phi_{t}^{E}\left({ }^{M} \widetilde{\varphi}_{t}^{i j}\right)^{2} \mu^{i j} H_{t}^{i} \mathrm{~d} t
\end{aligned}
$$

and

$$
\begin{aligned}
\log Z_{T}= & \int_{0}^{T} \sum_{i} \sum_{j}\left(\lambda_{t}^{M} \widetilde{\varphi}_{t}^{i j}-{ }^{L} \widetilde{\varphi}_{t}^{i j}\right) \mu^{i j} H_{t}^{i} \mathrm{~d} t \\
& +\int_{0}^{T} \sum_{i} \sum_{j} \log \left(1-\lambda_{t}^{M} \widetilde{\varphi}_{t}^{i j}+{ }^{L} \widetilde{\varphi}_{t}^{i j}\right) \mathrm{d} H_{t}^{i j}
\end{aligned}
$$

respectively. Equating the above equations while using the fact that $U_{t}^{i j}=H_{t}^{i j}-\int_{0}^{t} \mu^{i j} H_{s}^{i} \mathrm{~d} s$ and simplifying the terms results in (4).

\section{Finding a candidate for entropy minimization}

Given our price process in (1), we see that, for every $i \in \mathcal{y}$ and $i \neq j$,

$$
\begin{gathered}
{ }^{M} \widetilde{\varphi}_{t}^{i j}=\theta_{t}^{i j} X_{t-} \quad \text { and } \quad \lambda_{t}=\frac{\sum_{i} \eta_{t}^{i} H_{t}^{i}}{X_{t-} \sum_{i} \sum_{j}\left(\theta_{t}^{i j}\right)^{2} \mu^{i j} H_{t}^{i}} \\
\Longleftrightarrow \quad \lambda_{t} X_{t-}=\sum_{i} H_{t}^{i}\left(\frac{\eta_{t}^{i}}{\sum_{j}\left(\theta_{t}^{i j}\right)^{2} \mu^{i j}}\right)=: \sum_{i} H_{t}^{i} \hat{\lambda}_{t}(i, j),
\end{gathered}
$$


where

$$
\hat{\lambda}_{t}(i, j):=\frac{\eta_{t}^{i}}{\sum_{j}\left(\theta_{t}^{i j}\right)^{2} \mu^{i j}} .
$$

Here the equations are meant to be in the sense of indistinguishability of the respective processes. Note that $\sum_{i} \sum_{j}\left(\theta_{t}^{i j}\right)^{2} \mu^{i j} H_{t}^{i}<\infty$ due to the boundedness of $\theta^{i j}$ and the fact that $C$ has a finite state space. Also, note from above that $\theta^{i i}=0$. Equation (4) then reduces to

$$
\begin{aligned}
c^{E}= & -\int_{0}^{T} \sum_{i} \sum_{j} \lambda_{t} \phi_{t}^{E} X_{t-}^{2}\left(\theta_{t}^{i j}\right)^{2} \mu^{i j} H_{t}^{i} \mathrm{~d} t+\int_{0}^{T} \sum_{i} \sum_{j} \phi_{t}^{E} X_{t-} \theta_{t}^{i j} \mu^{i j} H_{t}^{i} \mathrm{~d} t \\
& -\int_{0}^{T} \sum_{i} \sum_{j}{ }^{L} \widetilde{\varphi}_{t}^{i j} \mu^{i j} H_{t}^{i} \mathrm{~d} t+\int_{0}^{T} \sum_{i} \sum_{j} \lambda_{t} X_{t-} \theta_{t}^{i j} \mu^{i j} H_{t}^{i} \mathrm{~d} t \\
& +\int_{0}^{T} \sum_{i} \sum_{j}\left\{\log \left(1-\lambda_{t} \theta_{t}^{i j} X_{t-}+{ }^{L} \widetilde{\varphi}_{t}^{i j}\right)-\theta_{t}^{i j} X_{t-} \phi_{t}^{E}\right\} \mathrm{d} H_{t}^{i j} .
\end{aligned}
$$

Define $\mathcal{C}_{b}:=\mathcal{C}_{b}([0, T] \times\{1, \ldots, m\})$, the space of continuous and bounded functions of $u:[0, T] \times\{1, \ldots, m\} \rightarrow \mathbb{R}, u_{t}:=u(t, \cdot): \mathcal{y} \rightarrow \mathbb{R}$, and $\Delta u_{t}:=u\left(t, C_{t}\right)-u\left(t, C_{t-}\right)$. We proceed with the ansatz that there exists a sufficiently smooth function $u \in \mathcal{C}_{b}$ such that, upon transition from $i$ to $j$ at time $t$,

$$
\log \left(1-\lambda_{t} \theta_{t}^{i j} X_{t-}+{ }^{L} \widetilde{\varphi}_{t}^{i j}\right)-\theta_{t}^{i j} X_{t-} \phi_{t}^{E}=u(t, j)-u(t, i) .
$$

With this ansatz, observe that we can write

$$
\begin{aligned}
& \sum_{0<t \leq T} \sum_{i} \sum_{j}\left\{\log \left(1-\lambda_{t} \theta_{t}^{i j} X_{t-}+{ }^{L} \tilde{\varphi}_{t}^{i j}\right)-\theta_{t}^{i j} X_{t-} \phi_{t}^{E}\right\} \Delta H_{t}^{i j} \\
& =\sum_{0<t \leq T}\left\{u\left(t, C_{t}\right)-u\left(t, C_{t-}\right)\right\} .
\end{aligned}
$$

Furthermore, we set

$$
u(T, \cdot)=0 \quad \text { on } y \text {. }
$$

We postulate that $\phi_{t}^{E} X_{t-}$ takes the form $\sum_{i} H_{t}^{i} \hat{\phi}_{t}(i, j):=\phi_{t}^{E} X_{t-}$, so that (4) can be recast as

$$
\begin{aligned}
c^{E}+u\left(0, C_{0}\right)= & -\int_{0}^{T} \sum_{i} \hat{\phi}_{t}(i, j) \eta_{t}^{i} H_{t}^{i} \mathrm{~d} t+\int_{0}^{T} \sum_{i} \sum_{j} \hat{\phi}_{t}(i, j) \theta_{t}^{i j} \mu^{i j} H_{t}^{i} \mathrm{~d} t \\
& -\int_{0}^{T} \sum_{i} \sum_{j}{ }^{L} \widetilde{\varphi}_{t}^{i j} \mu^{i j} H_{t}^{i} \mathrm{~d} t+\int_{0}^{T} \sum_{i} \sum_{j} \hat{\lambda}_{t}(i, j) \theta_{t}^{i j} \mu^{i j} H_{t}^{i} \mathrm{~d} t \\
& -\int_{0}^{T} \sum_{i} H_{t}^{i} \frac{\partial}{\partial t} u(t, i) \mathrm{d} t .
\end{aligned}
$$

Observe that, for the right-hand side of (7) to be constant, a possible solution might be to require that

$$
\frac{\partial}{\partial t} u(t, i)+\hat{\phi}_{t}(i, j) \eta_{t}^{i}-\sum_{j}\left\{\hat{\lambda}_{t}(i, j) \theta_{t}^{i j}+\hat{\phi}_{t} \theta_{t}^{i j}-{ }^{L} \widetilde{\varphi}_{t}^{i j}\right\} \mu^{i j}=0,
$$


together with (6) and the fact that

$$
c^{E}=-u\left(0, C_{0}\right)
$$

We further introduce

$$
g^{i}\left(t, u_{t}\right):=\hat{\phi}_{t}(i, j) \eta_{t}^{i}-\sum_{j}\left\{\hat{\lambda}_{t}(i, j) \theta_{t}^{i j}+\hat{\phi}_{t}(i, j) \theta_{t}^{i j}-{ }^{L} \widetilde{\varphi}_{t}^{i j}\right\} \mu^{i j},
$$

and arrive at a system of coupled ordinary differential equations for $u$ of the form

$$
\frac{\partial}{\partial t} u(t, i)+g^{i}\left(t, u_{t}\right)=0, \quad u(T, i)=0 \quad \text { for every } i \in \mathcal{Y} .
$$

We further find using our ansatz that, for $i \neq j$,

$$
{ }^{L} \widetilde{\varphi}^{i j}=\exp \left\{u(\cdot, j)-u(\cdot, i)+\hat{\phi}(i, j) \theta^{i j}\right\}+\hat{\lambda}(i, j) \theta^{i j}-1 .
$$

Replacing ${ }^{L} \widetilde{\varphi}_{t}^{i j}$ in (5) culminates in the following condition for $\hat{\phi}$ :

$$
X_{t-} \sum_{i} H_{t}^{i}\left\{\eta_{t}^{i}+\sum_{j} \theta_{t}^{i j}\left(\exp \left\{u(t, j)-u(t, i)+\hat{\phi}_{t}(i, j) \theta_{t}^{i j}\right\}-1\right) \mu^{i j}\right\} \mathrm{d} t=0
$$

for all $t \in[0, T]$. We see that the above is true if the following holds:

$$
\eta^{i}+\sum_{j} \theta^{i j}\left(\exp \left\{u(\cdot, j)-u(\cdot, i)+\hat{\phi}(i, j) \theta^{i j}\right\}-1\right) \mu^{i j}=0 \quad \text { for every } i \in \mathcal{y} .
$$

We shall call (9) the $\overline{\mathbb{Q}}$-martingale equation. Hence, any strategy $\phi^{E}$ that fulfills the $\overline{\mathbb{Q}}$-martingale equation is a potential candidate for the minimal entropy martingale measure.

\subsection{Existence of solutions}

Let us discuss the existence of $\hat{\phi}$ in (9). We present the following result.

Lemma 2. Let $u \in \mathcal{C}_{b}$. Then there is a bounded function $\hat{\phi}:[0, T] \times y \times y \rightarrow \mathbb{R}$ with $\hat{\phi}_{t}(i, j):=\hat{\phi}(t, i, j)$ which solves the martingale equation (9). Note also that $\theta^{i i}=0$.

Proof. Let

$$
\begin{aligned}
& f_{1}(\phi):=\eta_{t}^{i} \quad \text { for a fixed } i \in \mathcal{Y}, \\
& f_{2}(\phi):=-\sum_{j} \theta_{t}^{i j}\left(\exp \left\{u(t, j)-u(t, i)+\phi \theta_{t}^{i j}\right\}-1\right) \mu^{i j}
\end{aligned}
$$

Note that $f_{2}^{\prime}(\phi)=-\sum_{j}\left(\theta_{t}^{i j}\right)^{2}\left(\exp \left\{u(t, j)-u(t, i)+\phi \theta_{t}^{i j}\right\}-1\right) \mu^{i j}<0$, that is, $f_{2}$ is decreasing in $\phi$. For any $t \in[0, T]$, we consider the following three cases.

Case 1: all $\theta_{t}^{i j}>0$ for every $i \in \mathcal{y}$. Note that $f_{2}(0)=-\sum_{j} \theta_{t}^{i j}\left(\mathrm{e}^{u(t, j)-u(t, i)}-1\right) \mu^{i j}$, which can be either less than 0 or greater than $0, f_{2}(\infty)=-\infty$, and $f_{2}(-\infty)=$ $+\sum_{j} \theta_{t}^{i j} \mu^{i j}>0$.

Case 2: at least one $\theta_{t}^{i j}$ for $i \in \mathcal{Y}, i \neq j$, is greater than 0 with the others satisfying $-1<$ $\theta_{t}^{i j}<0$. Note that $f_{2}(0)=-\sum_{j} \theta_{t}^{i j}\left(\mathrm{e}^{u(t, j)-u(t, i)}-1\right) \mu^{i j}$, which can be either less than 0 or greater than $0, f_{2}(\infty)=-\infty$, and $f_{2}(-\infty)=+\infty$. 
Case 3: $-1<\theta_{t}^{i j}<0$ for every $i \in \mathcal{y}$. Note that $f_{2}(0)=-\sum_{j} \theta_{t}^{i j}\left(\mathrm{e}^{u(t, j)-u(t, i)}-1\right) \mu^{i j}$, which can be either less than 0 or greater than $0, f_{2}(\infty)=+\sum_{j} \theta_{t}^{i j} \mu^{i j}<0$, and $f_{2}(-\infty)=+\infty$.

Hence, we see that, for any $t \in[0, T]$, there exists $\phi=: \hat{\phi}_{t}^{i j} \in \mathbb{R}$ such that $f_{1}(\hat{\phi})=f_{2}(\hat{\phi})$ for all three cases if

$$
\eta_{t}^{i} \in[\underline{\phi}, \bar{\phi}]
$$

where

$$
-\sum_{j}\left|\theta_{t}^{i j}\right| \mu^{i j}<\underline{\phi} \leq \bar{\phi}<+\sum_{j}\left|\theta_{t}^{i j}\right| \mu^{i j}
$$

In other words, existence is guaranteed if we choose $\eta_{t}^{i}$ to be bounded away from $-\sum_{j}\left|\theta_{t}^{i j}\right| \mu^{i j}$ and $+\sum_{j}\left|\theta_{t}^{i j}\right| \mu^{i j}$ for any $t \in[0, T]$. Furthermore, if (10) is respected, it is clear that the function $\phi_{t}(i, j)$ is bounded. Also, from (9) we see that $\hat{\phi}_{t}(i, j)$ depends on $i$ and $j$, and, for a fixed $i, \hat{\phi}_{t}(i, j)$ will remain the same for every $j$. We now define $\hat{\phi}_{t}(i, j)$ as the unique function that solves the $\overline{\mathbb{Q}}$-martingale equation (9).

The proof of the next proposition follows very similar lines as in the proofs of Lemma 3.5 and Theorem 3.8 of [20] or as in the appendix of [13], and so we omit it.

Proposition 3. There exists a unique solution $\hat{u} \in \mathcal{C}_{b}$ which solves (8).

\section{Entropy minimizer for chains}

Prior to stating the main theorem, we have the following result.

Proposition 4. Let $\widetilde{a}^{i j}, i, j \in \mathcal{Y}, i \neq j$, be a family of real-valued, bounded $\mathbb{F}$-predictable processes such that $\tilde{a}^{i j}>-1$ with $\tilde{a}^{i i}=\widetilde{a}^{m}=0$ for $i=1, \ldots, m$. Then

$$
\begin{aligned}
\mathbb{E}_{\mathbb{P}}\left[\exp \left\{\int_{0}^{t} \sum_{i} \sum_{j} \log \left(1+\tilde{a}_{s}^{i j}\right) \mathrm{d} H_{s}^{i j}\right\}\right] & \leq \exp \left\{\sup _{s \leq t, i, j \in \mathcal{Y}}\left\|\tilde{a}_{s}^{i j}\right\|_{L^{\infty}} \sum_{i} \sum_{j} \mu^{i j}\right\} \\
& <\infty
\end{aligned}
$$

Proof. Let $M^{\prime}=\int \sum_{i} \sum_{j} \tilde{a}^{i j} \mathrm{~d} U^{i j}$. Then $M^{\prime}$ is a local martingale. Let us now consider the stochastic differential equation

$$
\mathrm{d} Z_{t}^{\prime}=Z_{t-}^{\prime} \mathrm{d} M_{t}^{\prime}, \quad Z_{0}^{\prime}=1
$$

Its solution is given by

$$
Z_{t}^{\prime}=\exp \left\{-\int_{0}^{t} \sum_{i} \sum_{j} \widetilde{a}_{s}^{i j} \mu^{i j} H_{s}^{i} \mathrm{~d} s\right\} \exp \left\{\int_{0}^{t} \sum_{i} \sum_{j} \log \left(1+\widetilde{a}_{s}^{i j}\right) \mathrm{d} H_{s}^{i j}\right\} .
$$

Note that $Z_{-}^{\prime}$ is an adapted process which is left continuous with right limits, so $Z_{-}^{\prime}$ is locally bounded (see [19, p. 166]). Since $Z_{-}^{\prime}$ is locally bounded, $Z^{\prime}$ is a local martingale since it is, by (11), the stochastic integral with respect to $M^{\prime}$ which is a local martingale (see Theorem 29 of [19, Chapter IV]). Observe that $Z^{\prime}$ is nonnegative, and by Fatou's lemma we can conclude that 
$Z^{\prime}$ is a supermartingale. Hence,

$$
\begin{aligned}
1= & \mathbb{E}_{\mathbb{P}}\left[Z_{0}^{\prime}\right] \\
\geq & \mathbb{E}_{\mathbb{P}}\left[Z_{t}^{\prime}\right] \\
= & \mathbb{E}_{\mathbb{P}}\left[\exp \left\{-\int_{0}^{t} \sum_{i} \sum_{j} \widetilde{a}_{s}^{i j} \mu^{i j} H_{s}^{i} \mathrm{~d} s+\int_{0}^{t} \sum_{i} \sum_{j} \log \left(1+\widetilde{a}_{s}^{i j}\right) \mathrm{d} H_{s}^{i j}\right\}\right] \\
\geq & \exp \left\{-\left(\sup _{s \leq t, i, j \in \mathcal{Y}}\left\|\widetilde{a}_{s}^{i j}\right\|_{L^{\infty}} t \sum_{i} \sum_{j} \mu^{i j}\right)\right\} \\
& \times \mathbb{E}_{\mathbb{P}}\left[\exp \left\{\int_{0}^{t} \sum_{i} \sum_{j} \log \left(1+\tilde{a}_{s}^{i j}\right) \mathrm{d} H_{s}^{i j}\right\}\right] .
\end{aligned}
$$

Rearranging yields the result.

We now state the main theorem.

Theorem 5. ( $\mathbb{Q}^{E}$ for chains.) Let $\eta_{t}^{i} \in[\phi, \bar{\phi}]$. Then the process $Z=\left(Z_{t}\right)$ defined by

$$
Z_{t}=\mathcal{E}\left(\int_{0}^{t} \sum_{i} \sum_{j}\left[\exp \left\{\Delta \hat{u}_{s}+\hat{\phi}_{s}(i, j) \theta_{s}^{i j}\right\}-1\right] \mathrm{d} U_{s}^{i j}\right)
$$

is the density process of the entropy minimizing martingale measure.

Proof. Let us now carry out the verifications as outlined in Section 3.1.

Step 1: $\overline{\mathbb{Q}}$ is an equivalent probability measure. We will use the criterion of Theorem III.1. of [14]. Let the local martingale $N$ be defined as

$$
N:=-\int \lambda \mathrm{d} M+L=\int \sum_{i} \sum_{j}\left({ }^{L} \widetilde{\varphi}^{i j}-\lambda^{M} \widetilde{\varphi}^{i j}\right) \mathrm{d} U^{i j}
$$

with

$$
N_{t}=\int_{0}^{t} \sum_{i} \sum_{j}\left(\exp \left\{\Delta \hat{u}_{s}+\hat{\phi}_{s}(i, j) \theta_{s}^{i j}\right\}-1\right) \mathrm{d} U_{s}^{i j}
$$

since $\hat{\phi}$ and $\theta$ are bounded, $N$ is locally bounded. Also, observe that

$$
\Delta N_{t}=\sum_{i} \sum_{j}\left(\exp \left\{\Delta \hat{u}_{t}+\hat{\phi}_{t}(i, j) \theta_{t}^{i j}\right\}-1\right) \Delta H_{t}^{i j},
$$

so that $\Delta N>-1$ since $\mathrm{e}^{x}>0$ for all $x$. We now seek to find the $\mathbb{P}$-compensator of $U$ which we shall denote by $B$. Note that

$$
U_{t}=\int_{0}^{t} \sum_{i} \sum_{j}\left(\hat{\phi}_{s}(i, j) \theta_{s}^{i j} \exp \left\{\Delta \hat{u}_{s}+\hat{\phi}_{s}(i, j) \theta_{s}^{i j}\right\}-\exp \left\{\Delta \hat{u}_{s}+\hat{\phi}_{s}(i, j) \theta_{s}^{i j}\right\}+1\right) \mathrm{d} H_{s}^{i j}
$$

To show that $U$ admits a predictable compensator, note that

$$
\int_{0}^{t} \sum_{i} \sum_{j}\left|\left(\hat{\phi}_{s}(i, j) \theta_{s}^{i j} \exp \left\{\Delta \hat{u}_{s}+\hat{\phi}_{s}(i, j) \theta_{s}^{i j}\right\}-\exp \left\{\Delta \hat{u}_{s}+\hat{\phi}_{s}(i, j) \theta_{s}^{i j}\right\}+1\right)\right| \mu^{i j} H_{s}^{i} \mathrm{~d} s<\infty
$$

due to the boundedness of $\theta^{i j}, \hat{\phi}(i, j)$, and $\hat{u}$. From Theorem II.1.28 of [11], this implies the 
integrability of

$$
\hat{\phi}_{s}(i, j) \theta_{s}^{i j} \exp \left\{\Delta \hat{u}_{s}+\hat{\phi}_{s}(i, j) \theta_{s}^{i j}\right\}-\exp \left\{\Delta \hat{u}_{s}+\hat{\phi}_{s}(i, j) \theta_{s}^{i j}\right\}+1=: f_{s}^{i j}
$$

with respect to $U^{i j}$ and

$$
\int_{0}^{t} \sum_{i} \sum_{j} f_{s}^{i j} \mathrm{~d} U_{s}^{i j}=\int_{0}^{t} \sum_{i} \sum_{j} f_{s}^{i j} \mathrm{~d} H_{s}^{i j}-\int_{0}^{t} \sum_{i} \sum_{j} f_{s}^{i j} \mu^{i j} H_{s}^{i} \mathrm{~d} s .
$$

Hence, the $\mathbb{P}$-compensator $A$ of $U$ is given by

$$
A_{t}:=\int_{0}^{t} \sum_{i} \sum_{j} f_{s}^{i j} \mu^{i j} H_{s}^{i} \mathrm{~d} s .
$$

It is easily seen that $\mathbb{E}_{\mathbb{P}}\left[\exp \left\{A_{T}\right\}\right]<\infty$ due to the boundedness of the functions $\Delta \hat{u}, \hat{\phi}$, and $\theta$ and the fact that there are a finite number of states for the chain.

Step 2: $\overline{\mathbb{Q}}$ is a martingale measure. Note that ${ }^{M} \widetilde{\varphi}^{i j}$ is bounded. Also, $L$ and $[M, L]$ are locally bounded. Hence, due to Corollary $1, \overline{\mathbb{Q}}$ is a martingale measure.

Step 3: $H(\overline{\mathbb{Q}}, \mathbb{P})<\infty$. Recall that the density $Z=\mathrm{d} \overline{\mathbb{Q}} / \mathrm{d} \mathbb{P}$ may be expressed as

$$
Z_{T}=\frac{\mathrm{d} \overline{\mathbb{Q}}}{\mathrm{dP}}=\exp \left\{c^{E}+\int_{0}^{T} \frac{\sum_{i} H_{t}^{i} \hat{\phi}_{t}(i, j)}{X_{t-}} \mathrm{d} X_{t}\right\} .
$$

Let us evaluate

$$
\begin{aligned}
\mathbb{E}_{\overline{\mathbb{Q}}}\left[\int_{0}^{T} \sum_{i} \sum_{j} \hat{\phi}_{t}(i) \theta_{t}^{i j} \mathrm{~d} U_{t}^{i j}\right]= & \mathbb{E}_{\overline{\mathbb{Q}}}\left[\int_{0}^{T} \sum_{i} \hat{\phi}_{t}(i)\left(-\eta_{t}^{i}+\sum_{j} \theta_{t}^{i j} \mu^{i j}\right) H_{t}^{i} \mathrm{~d} t\right] \\
& -\mathbb{E}_{\overline{\mathbb{Q}}}\left[\int_{0}^{T} \sum_{i} \sum_{j} \hat{\phi}_{t}(i) \theta_{t}^{i j} \mu^{i j} H_{t}^{i} \mathrm{~d} t\right] \\
= & -\mathbb{E}_{\overline{\mathbb{Q}}}\left[\int_{0}^{T} \sum_{i} \hat{\phi}_{t}(i) \eta_{t}^{i} H_{t}^{i} \mathrm{~d} t\right],
\end{aligned}
$$

where (12) is obtained by substituting the $\overline{\mathbb{Q}}$-martingale condition of (9). Finally, we obtain

$$
H(\overline{\mathbb{Q}}, \mathbb{P})=\mathbb{E}_{\overline{\mathbb{Q}}}\left[c^{E}\right]<\infty .
$$

Step 4: $\int \phi^{E} \mathrm{~d} S$ is a true $\mathbb{Q}$-martingale for all $\mathbb{Q} \in \mathcal{M}^{\mathrm{e}}$ with $H(\mathbb{Q}, \mathbb{P})<\infty$. We require that $\mathbb{E}_{\mathbb{P}}\left[\exp \left\{\beta \int_{0}^{T} \psi^{2} \mathrm{~d}[S, S]_{t}\right\}\right]<\infty$ for some $\beta>0$. We have $\psi_{t}=\sum_{i} H_{i}^{t} \hat{\phi}_{t}(i, j) / X_{t-}$, so that $\psi_{t}^{2}=\sum_{i} H_{t}^{i} \hat{\phi}_{t}^{2}(i, j) / X_{t-}^{2}$. We also have, for $i \neq j, \mathrm{~d}[S, S]_{t}=X_{t-}^{2} \sum_{i} \sum_{j}\left(\theta_{t}^{i j}\right)^{2} \mathrm{~d} H_{t}^{i j}$. We now have

$$
\begin{aligned}
& \mathbb{E}_{\mathbb{P}}\left[\exp \left\{\beta \int_{0}^{T} \psi^{2} \mathrm{~d}[S, S]_{t}\right\}\right] \\
& \leq \mathbb{E}_{\mathbb{P}}\left[\exp \left\{\int_{0}^{T} \sum_{i} \sum_{j}\left(\theta_{t}^{i j}\right)^{2} \mathrm{~d} H_{t}^{i j}\right\}\right] \mathbf{1}_{\left\{\beta:=\left(\sup _{t \in[0, T], i \in \mathcal{Y}} 2\left\|\hat{\phi}_{t}^{2}(i, j)\right\|_{\infty}\right)^{-1}>0\right\}} \\
& \quad \leq \exp \left\{\sup _{t \in[0, T], i, j \in \mathcal{Y}}\left\|\mathrm{e}^{\left(\theta_{t}^{i j}\right)^{2}}\right\|_{\infty} T \sum_{i} \sum_{j} \mu^{i j}\right\} \\
& \quad<\infty
\end{aligned}
$$

due to the boundedness of $\theta_{t}^{i j}$ and $\mu^{i j}$ and by setting $\widetilde{a}_{t}^{i j}:=\mathrm{e}^{\left(\theta_{t}^{i j}\right)^{2}}-1$ in Proposition 4. 


\section{Comments on the $\mathbb{Q}^{E}$-Markov chain}

\subsection{Connection to the general results of Girsanov's theorem for Markov chains}

Now that the minimal entropy martingale measure for continuous-time Markov chains has been found, we would like to see how it can be connected to the general result of measure change for chains. The following result is from [22].

Theorem 6. Let the probability measure $\widetilde{\mathbb{P}}$ be defined by

$$
\frac{\mathrm{d} \widetilde{\mathbb{P}}}{\mathrm{d} \mathbb{P}}=\rho_{T} \quad \mathbb{P} \text {-a.s. }
$$

with Radon-Nikodym density

$$
\rho_{T}=1+\int_{0}^{T} \sum_{i} \sum_{j} \rho_{t-} \widetilde{\varphi}_{t}^{i j} \mathrm{~d} U_{t}^{i j},
$$

where $\widetilde{\varphi}$ is locally bounded and predictable for all $i, j \in \mathcal{y}$ and $\widetilde{\varphi}(i, i)=0$. Then the following statements hold.

(i) The process $C$ is an $\mathbb{F}$-Markov chain under $\widetilde{\mathbb{P}}$.

(ii) The transition rates $\widetilde{\Lambda}(t):=\left[\tilde{\mu}^{i j}(t)\right]_{i, j \in\{1, \ldots, m\}}$ for $C$ under $\widetilde{\mathbb{P}}$ for $i \neq j$ have the form

$$
\tilde{\mu}_{t}^{i j}:=\tilde{\mu}^{i j}(t)=\left(1+\widetilde{\varphi}_{t}^{i j}\right) \mu^{i j} \quad \text { for all } t \in[0, T] .
$$

Proof. See Theorem 22.4 (Girsanov's formula for chains) of [22].

From Theorem 5, the transition rates under the minimal entropy martingale measure $\mu_{i j}^{\mathbb{Q}^{E}}$ take the form

$$
\mu_{i j}^{\mathbb{Q}^{E}}(t)=\exp \left\{\hat{\phi}_{t}(i, j) \theta_{t}^{i j}+\hat{u}(t, j)-\hat{u}(t, i)\right\} \mu^{i j} \quad \text { for all } t \in[0, T] .
$$

\section{Pricing under the minimal entropy martingale measure, $\mathbb{Q}^{E}$}

In this section we investigate the pricing of derivatives when the market follows a continuoustime Markov chain under the minimal entropy martingale measure. To illustrate how we can utilize the minimal entropy martingale measure for pricing issues, we follow the steps outlined in Section 3.A of [17]. In this section let the stock price process $X$ and the money market account $B$ be given by

$$
\begin{aligned}
& X_{t}=X_{0} \exp \left\{\int_{0}^{t} \sum_{i}\left(\eta^{i}-\sum_{j} \theta^{i j} \mu^{i j}\right) H_{s}^{i} \mathrm{~d} s+\int_{0}^{t} \sum_{i} \sum_{j} \log \left(\theta^{i j}+1\right) \mathrm{d} H_{s}^{i j}\right\}, \\
& B_{t}=\exp \left\{\int_{0}^{t} \sum_{i} H_{s}^{i} r^{i} \mathrm{~d} s\right\},
\end{aligned}
$$

where $\eta^{i}$ and $\theta^{i j}>-1$ are constants for a fixed $i \in \mathcal{Y}$, and the $r^{i}$ are constants that denote the statewise interest rates for $i=1, \ldots, m$. For $t \in[0, T]$, let $V_{t}$ denote the $\mathbb{Q}^{E}$-risk neutral price of a claim paying $G$ at time $T$, where $G$ is of the form $G=g^{C_{T}}\left(X_{T}\right)=\sum_{i} H_{T}^{i} g^{i}\left(X_{T}\right)$. Hence,

$$
V_{t}=\mathbb{E}_{\mathbb{Q}^{E}}\left[\exp \left\{-\int_{t}^{T} \sum_{i} H_{s}^{i} r^{i} \mathrm{~d} s\right\} G \mid \mathcal{F}_{t}\right] .
$$


We have

$$
X_{T}=X_{t} \exp \left\{\int_{t}^{T} \sum_{i}\left(\eta^{i}-\sum_{j} \theta^{i j} \mu^{i j}\right) H_{s}^{i} \mathrm{~d} s+\int_{t}^{T} \sum_{i} \sum_{j} \log \left(\theta^{i j}+1\right) \mathrm{d} H_{s}^{i j}\right\} .
$$

Due to (14), we can rewrite (13) as

$$
V_{t}=\mathbb{E}_{\mathbb{Q}^{E}}\left[\exp \left\{-\int_{t}^{T} \sum_{i} H_{s}^{i} r^{i} \mathrm{~d} s\right\} g^{C_{T}}\left(X_{t} X_{T-t}\right) \mid \mathcal{F}_{t}\right] .
$$

Note that the relevant state variables involved in the conditional value (13) are $\left(t, X_{t}, C_{t}\right)$. This is due to (14) and the Markov property which states that, given the present value $C_{t}$, the future and the past are independent. Hence, we can rewrite (15) as

$$
V_{t}=\sum_{i} H_{t}^{i} \mathbb{E}_{\mathbb{Q}^{E}}\left[\exp \left\{-\int_{t}^{T} \sum_{i} H_{s}^{i} r^{i} \mathrm{~d} s\right\} g^{C_{T}}\left(X_{t} X_{T-t}\right) \mid C_{t}=i, X_{t}=x\right] .
$$

Define the statewise prices $w^{i}$ as

$$
w^{i}(t, x):=\mathbb{E}_{\mathbb{Q}^{E}}\left[\exp \left\{-\int_{t}^{T} \sum_{i} H_{s}^{i} r^{i} \mathrm{~d} s\right\} g^{C_{T}}\left(X_{t} X_{T-t}\right) \mid C_{t}=i, X_{t}=x\right] .
$$

Finally, we see that the price at time $t$ of the claim can be written as

$$
V_{t}=\sum_{i} H_{t}^{i} w^{i}\left(t, X_{t}\right)
$$

The discounted price process $\widetilde{V}_{t}=\left(\exp \left\{-\int_{0}^{t} \sum_{i} H_{s}^{i} r^{i} \mathrm{~d} s\right\} V_{t}\right)_{t \in[0, T]}$ is a $\mathbb{Q}^{E}$-martingale. We assume that the functions $w^{i}(t, x)$ are continuously differentiable so that Itô's formula can be applied. By the martingale property, the $\mathrm{d} t$ terms must vanish, giving us the partial differential equations

$$
\begin{aligned}
0= & -r^{i} w^{i}(t, x)+\frac{\partial}{\partial t} w^{i}(t, x)+\frac{\partial}{\partial x} w^{i}(t, x) x\left(\eta^{i}-\sum_{j} \theta^{i j} \mu^{i j}\right) \\
& +\sum_{j}\left(w^{j}\left(t, x\left(1+\theta^{i j}\right)\right)-w^{i}(t, x)\right) \mu_{i j}^{\mathbb{Q}^{E}},
\end{aligned}
$$

with boundary conditions

$$
w^{i}(x, T)=g^{i}(x), \quad i=1, \ldots, m .
$$

\subsection{On the applicability of Itô's lemma}

As indicated by Norberg [17], the assumption that the functions $w^{i}(t, x)$ are continuously differentiable does not typically hold true and the functions may even be discontinuous. This particular problem was then investigated by the same author and led to [18], in which he explored those points on the functions that are nonsmooth and constructed a numerical method with a controlled global error to solve the differential equations. In this paper we do not embark on this journey. 


\section{Conclusion}

We derived the minimal entropy martingale measure for continuous-time Markov chains where conditions for absence of arbitrage are spelled out. Nonarbitrage valuation of derivatives was discussed, where we demonstrated how the transition intensities under the minimal entropy martingale measure would appear within the integrodifferential equations for the arbitrage free price. An extension towards allowing absorbing states in the concrete market model could be a topic of further research.

\section{References}

[1] Aase, K. K. (1988). Contingent claims valuation when the security price is a combination of an Itô process and a random point process. Stoch. Process. Appl. 28, 185-220.

[2] Benth, F. E. AND Meyer-Brandis, T. (2005). The density process of the minimal entropy martingale measure in a stochastic volatility model with jumps. Finance Stoch. 9, 563-575.

[3] Björk, T., Kabanov, Y. And RungGaldier, W. (1997). Bond market structure in the presence of marked point processes. Math. Finance 7, 211-239.

[4] Carr, P., Geman, H., Madan, D. B. And Yor, M. (2001). The fine structure of asset returns: an empirical investigation. J. Business 75, 305-332.

[5] Delbaen, F. et al. (2002). Exponential hedging and entropic penalties. Math. Finance 12, 99-123.

[6] Dellacherie, C. and Meyer, P.-A. (1980). Probabilités et Potentiel, 2nd edn. Hermann, Paris.

[7] Fritelli, M. (2000). The minimal entropy martingale measure and the valuation problem in incomplete markets. Math. Finance 10, 39-52.

[8] Grandits, P. and Rheinländer, T. (2002). On the minimal entropy martingale measure. Ann. Prob. 30, 10031038.

[9] Hubalek, F. and Sgarra, C. (2006). Esscher transforms and the minimal entropy martingale measure for exponential Lévy models. Quant. Finance 6, 125-145.

[10] Hull, J. And White, A. (1988). The pricing of options on assets with stochastic volatility. J. Finance 42, 281-300.

[11] Jacod, J. And Shiryaev, A. N. (2003). Limit Theorems for Stochastic Processes, 2nd edn. Springer, Berlin.

[12] Last, G. And Brandt, A. (1995). Marked Point Processes on the Real Line. Springer, New York.

[13] LeE, Y. ANd Rheinländer, T. (2012). Optimal martingale measures for defaultable assets. Stoch. Process. Appl. 122, 2870-2884.

[14] LÉPIngle, D. ANd MÉmin, J. (1978). Sur l'intégrabilité uniforme des martingales exponentielles. Z. Wahrscheinlichkeitsth. 42, 175-203.

[15] Merton, R. C. (1976). Option pricing when underlying asset returns are discontinuous. J. Financial Econom. 3, 125-144.

[16] Miyahara, Y. (2000). Minimal relative entropy martingale measure of birth and death process. Discussion paper, Nagoya City University.

[17] Norberg, R. (2003). The Markov chain market. ASTIN Bull. 33, 265-287.

[18] Norberg, R. (2005). Anomalous PDEs in Markov chains: domains of validity and numerical solutions. Finance Stoch. 9, 519-537.

[19] Protter, P. E. (2004). Stochastic Integration and Differential Equations, 2nd edn. Springer, Berlin.

[20] Rheinländer, T. AND Steiger, G. (2006). The minimal entropy martingale measure for general BarndorffNielsen/Shephard models. Ann. Appl. Prob. 16, 1319-1351.

[21] Rheinländer, T. and Sexton, J. (2011). Hedging Derivatives (Advanced Ser. Statist. Sci. Appl. Prob. 15). World Scientific Publishing, London.

[22] Rogers, L. C. G. And Williams, D. (2000). Diffusions, Markov Processes, and Martingales, Vol. 1, 2nd edn. Cambridge University Press.

[23] Rolski, T., Schmidli, H., Schmidt, V. And Teugels, J. (1998). Stochastic Processes for Insurance and Finance. John Wiley, Chichester.

[24] Steiger, G. (2005). The optimal martingale measure for investors with exponential utility function. Doctoral Thesis, ETH Zürich. 